\title{
Dark baryons not in ancient halo white dwarfs ${ }^{\star}$
}

\author{
M. Crézé1,8, V. Mohan ${ }^{3}$, A. C. Robin² , C. Reylé2, H. J. Mc Cracken ${ }^{6}$, \\ J.-C. Cuillandre ${ }^{4}$, O. Le Fèvre ${ }^{5}$, and Y. Mellier, \\ 1 Université de Bretagne Sud, BP 573, 56017 Vannes Cedex, France \\ e-mail: Michel.Creze@univ-ubs.fr \\ 2 CNRS UMR6091, Observatoire de Besançon, BP 1615, 25010 Besançon Cedex, France \\ 3 UP State Observatory, Manora Peak, Nainital 263129, India \\ ${ }^{4}$ CFHT Corp., PO box 1597, Kamuela Hawaii 96783, USA \\ 5 Laboratoire d'astrophysique de Marseille, Observatoire de Marseille Provence, Université de Provence et CNRS, \\ BP 8, Traverse du Siphon, 13376 Marseille Cedex, France \\ 6 Institut d'Astrophysique de Paris, 98 bis boulevard Arago, 75014 Paris, France \\ 7 LERMA, Observatoire de Paris, 61 avenue de l'Observatoire, 75014 Paris, France \\ 8 PCC, Collège de France, 11 place Marcelin Berthelot, 75231 Paris Cedex 05, France
}

Received 22 March 2004 / Accepted 19 May 2004

\begin{abstract}
Having ruled out the possibility that stellar objects are the main contributor of the dark matter embedding galaxies, microlensing experiments cannot exclude the hypothesis that a significant fraction of the Milky Way dark halo might be made of MACHOs with masses in the range $0.5-0.8 M_{\odot}$. Ancient white dwarfs are generally considered the most plausible candidates for such MACHOs. We report the results of a search for such white dwarfs in a proper motion survey covering a $0.16 \mathrm{sq}$. deg. field at three epochs at high galactic latitude, and 0.938 sq. deg. at two epochs at intermediate galactic latitude (VIRMOS survey), using the $\mathrm{CFH}$ telescope. Both surveys are complete to $I=23$, with detection efficiency fading to 0 at $I=24$.2. Proper motion data are suitable to separate unambiguously halo white dwarfs identified as belonging to a non rotating system. No candidates were found within the colour-magnitude-proper motion volume where such objects can be safely discriminated from any standard population as well as from possible artefacts. In the same volume, we estimate the maximum white dwarf halo fraction compatible with this observation at different significance levels if the halo is at least 14 gigayears old and under different ad hoc initial mass functions. Our data alone rule out a halo fraction greater than $14 \%$ at a $95 \%$ confidence level. Combined with two previous investigations exploring comparable volumes, this pushes the limit below $4 \%$ ( $95 \%$ confidence level) or below $1 \%$ (64\% confidence), and implies that if baryonic dark matter is present in galaxy halos, it is not, or is only marginally in the form of faint hydrogen white dwarfs.
\end{abstract}

Key words. cosmology: dark matter - Galaxy: halo - stars: white dwarfs

\section{Introduction}

A non rotating or weakly rotating, temperature supported halo is what seems to best satisfy most existing requirements for the bulk of dark matter implied by the large-scale rotation curve of the Milky Way galaxy. If a more than marginal part of this dark matter is made of baryons, then it should be expected to appear in the form of moderately massive compact objects.

Concerning the identity of such objects, there were two kinds of candidates initially envisaged: Jupiter-like objects or brown dwarfs on the low mass side, very old fading degenerate stars (White Dwarfs) at larger masses $\left(0.5-0.8 M_{\odot}\right)$. Brown Dwarfs and planets were eliminated by early microlensing experiments (Alcock et al. 1997; Aubourg et al. 1993). Therefore cool white dwarfs appeared as the only possibility left (Charlot $\&$ Silk 1995). Chabrier (1999) and Chabrier et al. (2000b) show

* Based on observations made at Canada-France-Hawaii Telescope (CFHT). that ad hoc star formation scenarios are able to form an adequate number of such objects out of the protogalactic matter without producing unrealistic heavy element enrichment.

Second generation microlensing surveys at high galactic latitude rule out heavy halos fully made of very old white dwarfs (Aubourg et al. 1993). It is still not clear whether they imply some fraction of the halo in the form of massive compact halo objects (MACHOS). The EROS collaboration (Afonso et al. 2003) gives only an upper limit of about 25\% for masses ranging from 0.5 to $0.8 M_{\odot}$. The MACHO collaboration (Alcock et al. 1997) gives a range [20\%, 50\%] which seems to exclude fully non-baryonic halos, yet they admit that this result is somewhat model-dependent.

Direct detections of very ancient white dwarfs in colourproper motion surveys have now been obtained by different groups (Oppenheimer et al. 2001; Ibata et al. 2000; Ruiz \& Bergeron 2001; Mendez 2002), several of them with spectroscopic confirmation. However, in all positive cases, the 
sampling conditions were such that it is not possible to identify candidates unambiguously connected to the non-rotating component. Detected ancient white dwarfs are most likely members of the thick disc of the Milky Way (Reylé et al. 2001; Reid et al. 2001; Torres et al. 2002).

In contrast, deeper surveys explore regions of the colour-magnitude proper motion space where halo white dwarfs are the only expected contributors. In such surveys Ibata et al. (1999), Nelson et al. (2002), Goldman et al. (2002) do not report detections in these regions. The detections reported by Nelson et al. (2002) are not in the proper motion range where there is no ambiguity. Also, attempts to search for similar objects in Luyten's LHS and NLTT large proper motion surveys (Flynn et al. 2001) found none.

We report here on a ground-based effort to detect ancient halo white dwarfs from a deep photometry-proper motion survey (Sect. 2). The volume explored is 3 times that of the Nelson's field, almost the same order of magnitude as Goldman's. A colour-magnitude-proper motion criterion is established, isolating a volume where, based on a realistic range of galaxy models, the expected contributions of disc and thick disc components are negligible, while the detection efficiency is quite high. In this volume the expected contribution of halo models made of ancient white dwarfs is evaluated (Sect. 3). An estimation of the halo fraction in the form of cool hydrogen white dwarfs compatible with getting no detection in the observed sample is performed. Similar model predictions are produced for the observing conditions of previous investigators in order to produce a combined constraint on the halo fraction (Sect. 4 and conclusion).

\section{Survey description}

Observational data used in this investigation come from two different observing programs using wide field CCD cameras at the $3.60 \mathrm{~m}$ CFH Telescope at Mauna Kea (Hawaii). One program near the galactic pole (herein with SA57 field) in special selected area SA57 is the result of three observation campaigns by some of the authors of this paper. The other one is part of the so-called VIRMOS survey (Le Fèvre et al. 2004; McCracken et al. 2003). Both are almost equally deep.

\subsection{Field characteristics}

\subsubsection{VIRMOS field}

The VIRMOS survey uses the CFH12K camera. Out of the full VIRMOS survey fields, one subfield lying in the galactic anticenter direction $\left(\alpha_{2000}=2^{\mathrm{h}} 26^{\mathrm{m}}, \delta_{2000}=-4^{\circ} 30^{\prime}\right.$; $\left.l^{\mathrm{II}}=172^{\circ}, b^{\mathrm{II}}=-58^{\circ}\right)$ was observed on two epochs in 1999 and 2000 in $V$ and $I$ pass bands. The useful sky area covered is 0.938 sq. degree.

\subsubsection{SA57 field}

One north galactic pole field was observed in Selected Area $\operatorname{SA} 57\left(\alpha=13^{\mathrm{h}} 9^{\mathrm{m}} 5^{\mathrm{s}}, \delta=+29^{\circ} 9^{\prime} 30^{\prime \prime}\right)$. Observations were obtained during three observing runs (March 21-23, 1996,
April 23-25, 1998, May 27-29 2000). The UH8K camera, a mosaic of 8 CCDs each of $2048 \times 4096$ pixels was used during the first two runs. The CFH12K was used on the third run. In both cameras, the projected pixel size is 0.205 arcsec wide on the sky. The useful field is limited to the overlap between the three observing epochs, that is 0.16 square degrees. Series of 20 min exposures were obtained in the $V, R$ and $I$ filters. The total exposure time is $2 \mathrm{~h}$ in each filter at each epoch.

The seeing was better than 0.7 arcsec in 1998, about 0.9 in 1996 and about 0.8 in 2000. The 1998 run gives the best tool for star-galaxy separation and for faint object detection confirmation, but the completeness limit for proper motions is imposed by the 1996 run. In these conditions the completeness limit turns out to be somewhat below 25 in $V$ for extended lssources, as seen from the magnitude histogram. Completeness is expected slightly deeper for star-like sources. Full discussion of the detection efficiency issue for star like objects is given in Sects. 2.5 and 2.6.

\subsection{Source extraction and classification}

Source extraction was performed on the sum of all images at each epoch using the SExtractor package (Bertin \& Arnouts 1996). Associated with the source extraction, SExtractor provides a classification parameter named "class star" $(C L S)$ ranging from 0 (safely non-stellar) to 1 (safely star-like). This class is computed by a neural network which uses the distribution of flux between object pixels as input data and a library of simulated star and galaxy images as training set. Rather than replaying the training with a specific library, we preferred to cross check and calibrate the diagnostic on real images.

The resulting distribution of $C L S$ as a function of magnitude is plotted in Fig. 1, the VIRMOS field has been chosen for illustration. It is obvious from the histograms in the lower panel that at magnitudes brighter than $I=22.5$, the contamination of the high $C L S$ range by non-stellar objects is very marginal because there are not many galaxies.

The effect of the $C L S$ selection upon detection efficiency is investigated in Sect. 2.5. For deriving the accuracy of proper motions, (Sect. 2.3) non-stellar sources would heavily affect the statistics, hence we adopt a high $(C L S>0.8)$ selection threshold. On the contrary, when it comes to detecting as large a number of white dwarf candidates as possible, the detection efficiency must be maximum. Then the $C L S$ selection threshold for searching halo white dwarfs (Sect. 3), which in the present case amounts to delimitating the volume within which there are no white dwarfs detected, has been set to 0.4. According to Fig. 1 this threshold is sufficient to eliminate the overwhelming majority of obviously non stellar objects. Then we rely upon the proper motion criterion to eliminate misclassified galaxies.

\subsection{Astrometry}

While the SEXtractor package turns out to be an efficient source detection and classification tool, extensive tests and comparisons showed that the performances of the photometry and centering algorithm are not optimal for star-like sources. 

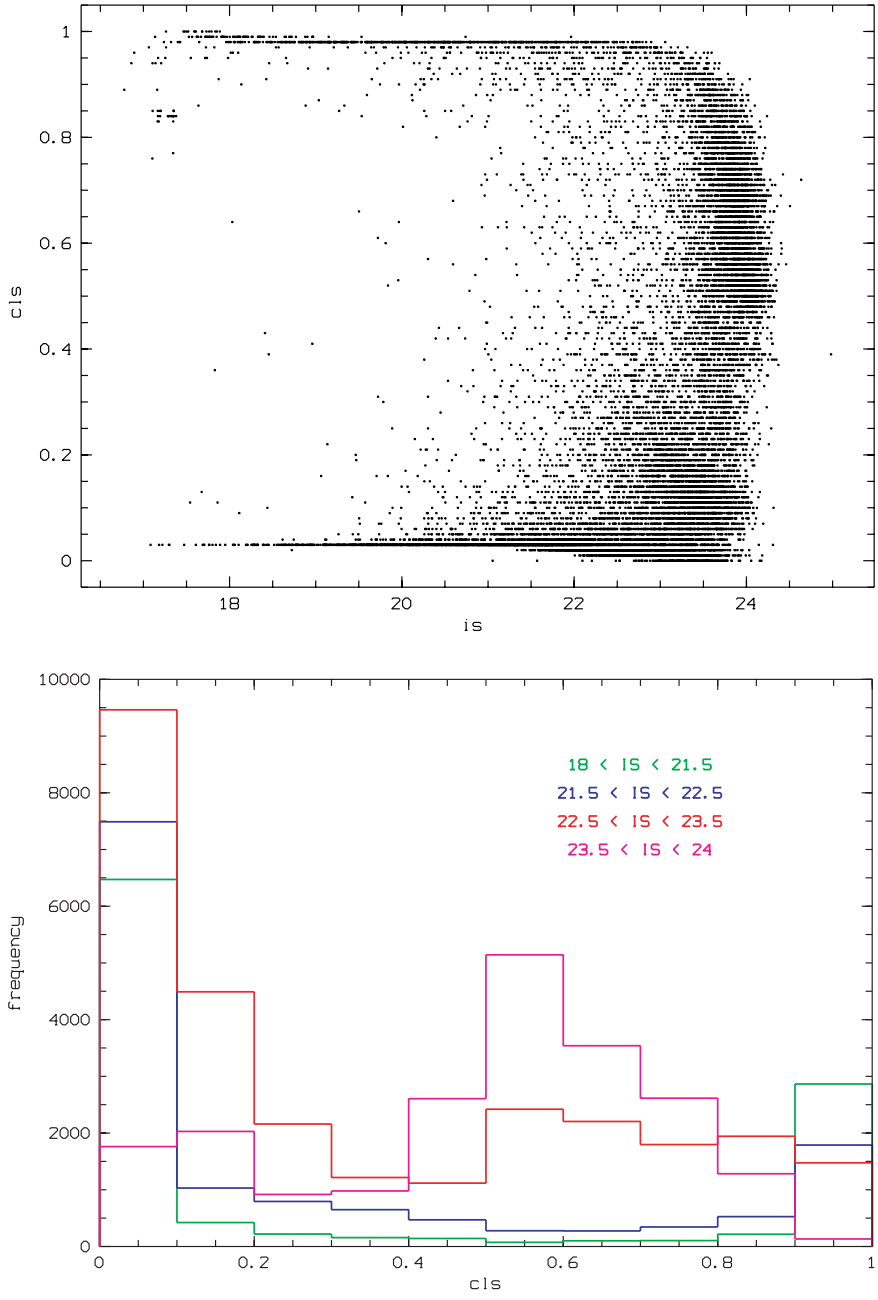

Fig. 1. Star galaxy separation: the $C L S$ classifier runs from 0 (safely non-stellar) to 1 (safely stellar). Top: $C L S$ is versus $I$ magnitude (in the Cousin's system). Bottom: $C L S$ frequency distributions in 4 mag ranges. Beyond magnitude 23.5 the galaxy peak at $C L S<0.1$ vanishes, indicating that the classifier no longer recognizes them.

So, the DAOPHOT/ALLSTAR package (Stetson 1987) was used to estimate magnitudes and relative $(x, y)$ positions on each CCD, in each band, at each epoch. Photometry and astrometry are obtained by fitting a variable empirical PSF separately in each CCD. It was also investigated whether the goodness of fit parameters provided by DAOPHOT would bring additional information concerning the star/galaxy separation. These attempts were not conclusive. The goodness of fit parameters are partially redundant with the neural network approach, with less discrimination power.

The astrometric accuracy can be assessed by the statistics of $x$ and $y$ differences between two epochs after correction of systematic effects related to colour and position detected in the VIRMOS field (see below).

To perform the statistics, objects with starlike profile at $C L S \geq 0.8$ have been selected to avoid accuracy degradation related to extended sources. Then the sample was split into 5 mag ranges each containing 500 sources or more. In order to avoid contamination of the statistics by real significant proper motions, a robust statistics based on the central part of the
Table 1. VIRMOS field, estimated standard errors of $\mathrm{d} x$ and $\mathrm{d} y$ differences (pixels) as a function of $I$ magnitude. Nos. is the number of sources used in the statistics.

\begin{tabular}{crrrrr}
\hline \hline$I$ mag & $18-20$ & $20-21$ & $21-22$ & $22-22.5$ & $22.5-23$ \\
\hline Nos. & 951 & 791 & 994 & 512 & 486 \\
$\sigma_{\mathrm{d} x}$ & 0.045 & 0.040 & 0.058 & 0.095 & 0.120 \\
$\sigma_{\mathrm{d} y}$ & 0.037 & 0.042 & 0.059 & 0.093 & 0.110 \\
\hline
\end{tabular}

histogram is derived from probability plots. The resulting standard errors are given in Table 1.

For all useful purposes the trend of $\mathrm{d} x, \mathrm{~d} y$ s.e. was approximated by the following formula (in pixel units):

$\sigma_{\mathrm{d} x}=0.04+0.05 * \exp \left(1.094 * I_{\mathrm{mag}}-24.0\right)$.

\subsubsection{VIRMOS proper motion data}

In the case of VIRMOS survey, the pipeline processing from which we got the data includes re-coordination of all the frames at each epoch in a single absolute astrometric reference frame. As a consequence, the reference to individual CCD pixels was lost. This turned out to generate various disturbances, each of little significance for the large scale galaxy survey which was the main goal of the VIRMOS collaboration. However for our proper motion purposes each effect deserved specific care.

- Due to the offset strategy between exposures we had to trace approximately regions of full overlap between individual CCDs in the composite epoch image. This restriction is responsible for reducing the proper motion survey from the nominal $1.2 \mathrm{sq}$. deg. down to an effective $0.938 \mathrm{sq}$. deg.

- Individual relative $(x, y)$ positions, as remeasured by the DAOPHOT PSF centering algorithm on each epoch image, reflected the possible distortions of the global reference frame. Distortion has been corrected a posteriori. We have obtained an estimation of the necessary correction by assuming that the average motion of a subsample dominated by remote objects is zero everywhere in the field. The estimation has been performed twice by computing the regressions $\Delta \mathrm{d} x=\mathrm{d} x(x, y)$ at order 3 in both $\mathrm{d} x$ and $\mathrm{d} y$. This was done separately:

a) on a subset of galaxy like objects selected on the basis having low $C L S$ but not being too extended;

b) on a selection of high $C L S$ sources with selection $19<$ $I<22$.

The pattern of systematic effects obtained from either data sets is quite the same. For the final data we choose the regression based on stellar image which is less dispersed. Proper motions were then corrected for these effects. The maximum correction across the field is less than 0.2 pixels in both $\mathrm{d} x$ and $\mathrm{d} y$. So it is not likely to disturb large proper motion investigations.

- Another distortion appears as a function of colour. It turns out to affect $\mathrm{d} x$ proper motion only. It is linear in $V-I$, its amplitude is about 0.15 pixels across the whole colour range. It also affects positions of galaxies as well as stellar 
sources. The effect has been corrected following exactly the same process as for the $x, y$ correction.

Due to its position in the anticenter direction, proper motion in longitude coincides almost exactly with the rotation component.

\subsubsection{SA57 proper motion data}

Relative proper motions are obtained by cross-matching DAOPHOT positions separately within each CCD between two epochs and independently in the 3 bands. Systematic displacements and distortions between two epochs are modelled by a second degree polynomial in $x$ and $y$, including cross term, for the 96-98 period. A third order polynomial has been necessary to cross-match 1998 UH8K data with 2000 CFH12K data. The polynomial coefficients are estimated under the assumption that the average position of moderately bright stellar objects has zero motion. A 3-sigma clipping is applied to eliminate proper motion stars. This gives a transform based on 70 to 100 reference stars $(19<V<23)$ in each CCD. Remote spheroid and thick disc population dominate reference stars. With this selection, we establish from our galaxy model that the zero of proper motions is roughly moving with a rotation lag of -0.007 arcsec per year along $x$ and -0.008 arcsec along $y$ with respect to the local standard of rest.

With three epochs it becomes possible to check for the possible existence of spurious proper motions which turn out to be frequent at faint magnitudes. It is not clear what mechanism generates such spurious proper motions. The most plausible explanation is a displacement of the photocenter of remote galaxies (not detected as galaxies by the $C L S$ criterion) presumably due to supernova events. Their number grows rapidly if we release the $C L S$ selection criterion. If motions are real, discrepancies between proper motions obtained between epoch 2 and epoch 1 on the one hand and between epochs 3 and 2 on the other hand must be consistent with each other within $3 \sigma$ of the point source position. Combining this criterion with a rather loose $C L S$ selection threshold ( $C L S>0.4$ at all epochs) provides bona fide high proper motion stars with minimum loss of detection efficiency.

Proper motions are first computed in $x$ and $y$ (the $y$ axis is along CCD columns). The 1998 alignment is chosen as reference. In this alignment $y$ is almost exactly the declination axis. The $\mu_{x}$ and $\mu_{y}$ components are then rotated to the $\left(\mu_{U}, \mu_{V}\right)$ axes (galactic radius, galactic rotation).

The magnitude-dependent standard error of star-like objects is estimated following the same process as in the case of the VIRMOS field. The trend of the proper motion s.e. with magnitude is suitably approximated by the following formula (in $\operatorname{arcsec} \operatorname{cen}^{-1}$ ):

$\sigma_{\mu}=0.74+\exp \left(1.58 * I_{\mathrm{mag}}-37.5\right)$

Eventually the proper motion limit for searching halo white dwarfs was set to $3 \sigma_{\mu}$.

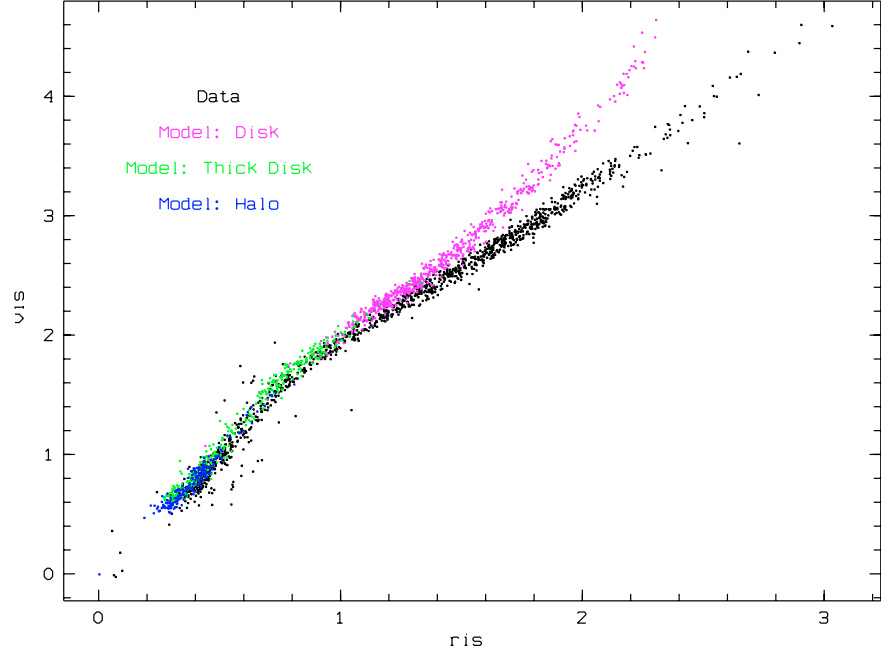

Fig. 2. $(V-I)$ vs. $(R-I)$ for data and model in the VIRMOS field. The plot is limited to bright stellar objects: $C L S>0.95,18<I<20$. Colours are black: data, blue: model (spheroid), green: (thick disc), magenta: (disc).

\subsection{Photometry}

As mentioned in the astrometry subsection, The DAOPHOT/ALLSTAR package was used to estimate magnitudes together with $(x, y)$ positions on each $C C D$, in each band, at each epoch. Photometry is performed by fitting a variable empirical PSF separately in each one of the CCDs of each mosaic.

\subsubsection{Random errors}

Statistical investigations (not reported here) of the photometric accuracy for stellar objects by comparing several exposures of identical fields at the same epoch suggest the following photometric error laws:

VIRMOS data:

$\sigma_{I}=0.02+\exp (-27+I)$

$\sigma_{V}=0.02+\exp (-27+V)$

SA57 data:

$\sigma_{I}=0.03+\exp (-26.1+0.958 I)$

$\sigma_{V}=0.03+\exp (-26.6+0.934 V)$.

\subsubsection{Systematics}

Although the observed photometric data are in principle tied to the standard Johnson-Cousins system, there is an obvious deviation of observed to theoretical colour-colour diagrams (the pattern is identical in both surveys) as can be seen in Fig. 2 . Model predictions are from Robin et al. (2003).

For the purpose of model-data comparisons, model colours were shifted in a way that forces the model sequence to fit the real data sequence. Assuming that:

- the shoulder point $(R-I)_{0},(V-I)_{0}$ at the junction between thick disc and disc is a fixed point. 


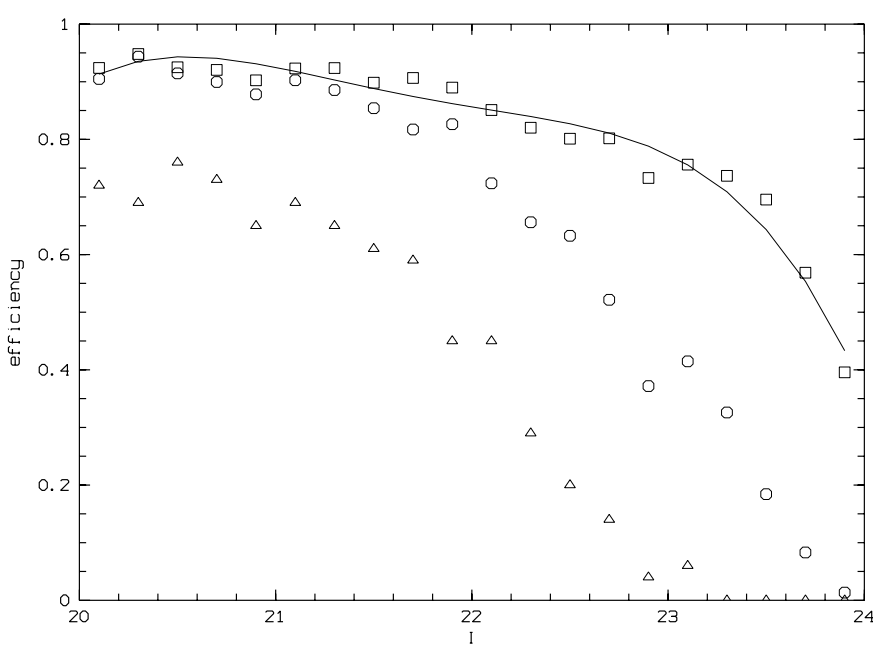

Fig. 3. Efficiency versus $I$ magnitude under various $C L S$ thresholds: 0.95 (triangles), 0.80 (circles), 0.40 (squares and fitted line).

- ends of sequences in data and model correspond to similar objects.

Colour conversions are then fitted so that the whole model sequence is brought into coincidence with the model.

In this way we expect to produce model predicted colours which may serve in interpreting the physical properties of real stars at least in a statistical way. This rather harsh procedure has only limited consequences on the present investigation since the change in $V-I$ is quite small. Anyway, there is almost no effect on the blue side, so our results concerning the photometry of ancient white dwarfs is essentially unaffected.

\subsection{Detection efficiency}

Numerical experiments are used for testing our capacity of identifying real stars out of these data. We seed the original frames with fake stellar images built on the observed PSF. The tool for this simulation is the ADDSTAR program of the DAOPHOT package. Then images are reprocessed and analysed through the complete chain. And detection efficiency is established as the fraction of fake stars seeded into the images eventually retrieved and selected at a given $C L S$ level.

Up to 2700 stellar images with $I$ magnitude ranging between 20 and 24 have been seeded into 9 different CCD frames distributed over the whole VIRMOS field at two epochs. Figure 3 shows the fraction of positive detections.

Efficiencies at $C L S$ threshold 0.4 as approximated by the regression curve appearing on the figure are used in Sect. 3 to establish the probability for any model predicted halo white dwarf to be detected in this survey.

\section{Halo white dwarfs}

A compromise had to be found between adopting a very high $C L S$ selection threshold which would warrant almost certainly stellar candidates at the expense of very low efficiency at faint magnitudes and low threshold which, at faint magnitudes, would let the sample be dominated by non-stellar objects with

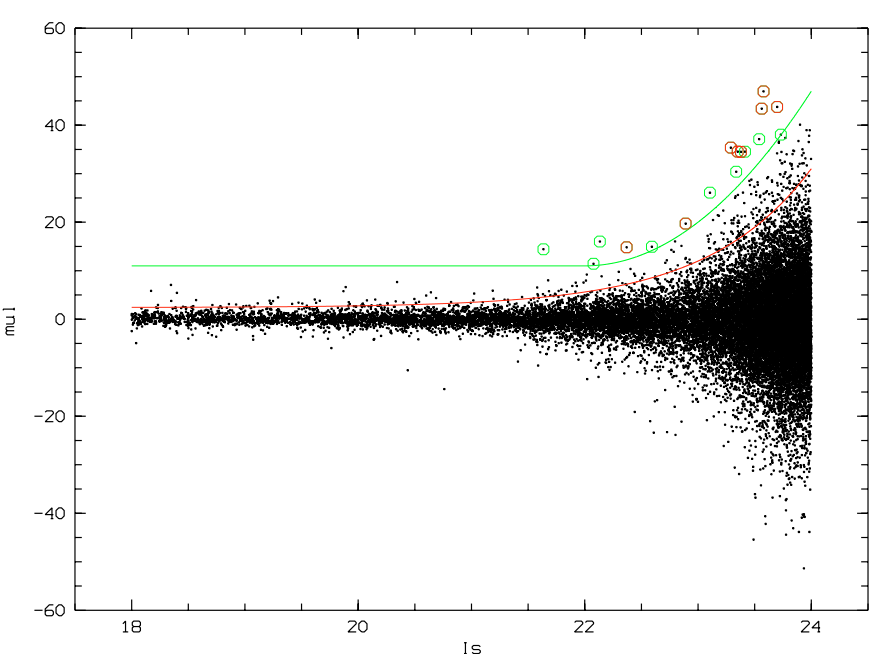

Fig. 4. Proper motion in longitude versus $I$ magnitude, observed data (VIRMOS field). The green limit is the upper envelope of possibly significant proper motions. Below this limit proper motions due to unrecognised non-stellar sources may contaminate. Circled dots are visually checked objects. All are identified as plate defects or misclassified galaxies. The red line is the $3 \sigma$ limit of the proper motion errors as estimated from Table 1.

the prospect of some of them producing artifactual proper motions resulting from extragalactic source variability. As a result of the detection efficiency study (Sect. 2.6), and of the $C L S /$ magnitude statistics (Fig. 1), it turns out that a $C L S>0.4$ at two epochs achieves a minimum loss of efficiency while letting in only a small fraction of obvious extended sources.

Above magnitude $I=22.5$ the only way to eliminate galaxies without eliminating most stellar objects is offered by proper motions, yet at faint magnitudes some artifactual proper motions appear, most related to uncorrected edge defects others to pixel defects, some possibly due to supernovae which would displace the photocenter at one epoch. Since we have only two epochs in the VIRMOS field there is no way to check for the later, while the former can occasionally be disregarded on the basis of a careful examination of images.

\subsection{Implementing a high proper motion limit in data}

Figure 4 is a plot of $\mu_{l}$ proper motion against $I$ for selected stellar candidates in the VIRMOS field. The zero of proper motions corresponds to the local standard of rest. Due to the rotation lag, the majority of nearby halo stars are expected to appear in the upper half of this diagram.

Then, we establish an empirical limit (in green on the $\mu_{l}$ versus $I$ plot) which is the upper envelope of proper motions in galactic longitude. There are seventeen objects found slightly above this line. Careful examination on CCD images of these objects above the limit shows that all of them correspond to image defects on one epoch and/or elongated image.

The four faintest borderline objects appearing in the VIRMOS field are too faint for clear discrimination on available CFHT images. They were submitted for spectroscopic observations at the VLT. Actually, preliminary VLT identification 


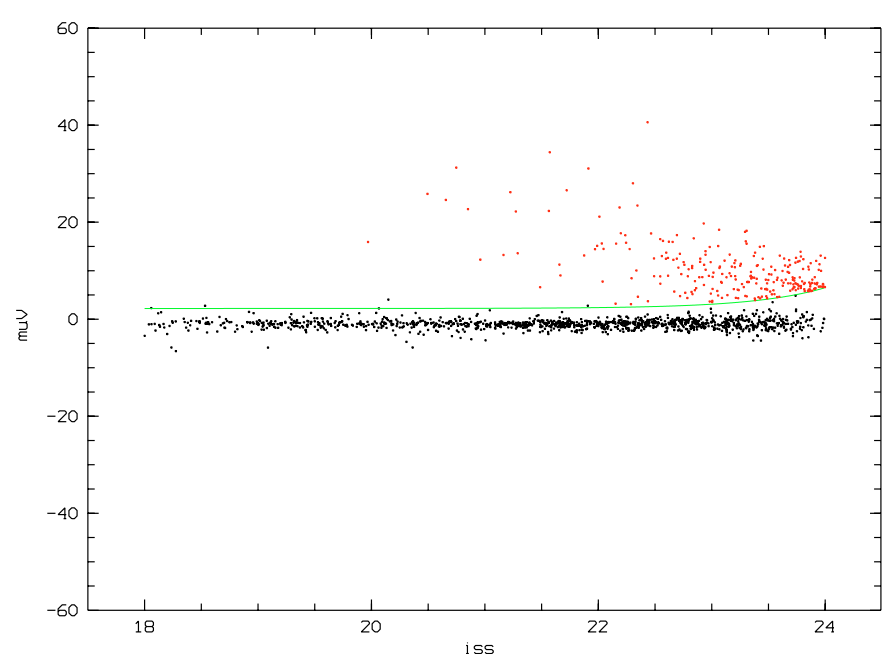

Fig. 5. Proper motion component along galaxy rotation versus $I$ magnitude. Observed data in Sa57 field (black dots). The green limit is the $3 \sigma$ of pointlike source proper motions. Red dots are halo white dwarfs from model $12 \mathrm{Gyr}$, IMF2 predicted above this limit.

frames, used as a third observation epoch, were sufficient to establish that the high proper motions were all spurious.

So there are no proper motion objects detectable above the green limit. As can be seen this limit is far above the 3 sigma limit of proper motion random errors as derived from point-like sources (in red).

Data in the SA57 field (Fig. 5) are much easier to interpret: with three observation epochs, spurious proper motions of undiscriminated non-stellar sources are efficiently detected. The green limit is simply the $3 \sigma$ limit of stellar source proper motions. There are a few real large proper motions at magnitudes brighter than $I=22$. The magnitudes, colours and proper motions of these stars are well compatible with being members of standard disc, thick disc and spheroid populations. Modelpredicted halo white dwarfs of similar magnitudes would have much larger proper motions.

\subsection{Models for dark halo white dwarfs}

Four different models of hydrogen halo white dwarf populations have been generated assuming halo ages of 12 or 14 Gyr. White dwarfs are created assuming two different initial mass functions and moved to present day colours following cooling lines by Chabrier et al. (2000a). These models do include $\mathrm{H} 2$ collisionally-induced absorption in the atmosphere, based on the atmosphere models of Saumon \& Jacobson (1999).

The parameters of the dark halo adopted for simulation purposes are according to Robin et al. (2003). The density law is a power law with an exponent of 2.44 and a flattening of 0.75 . The number of simulated white dwarfs should not depend too much on these parameters since they are detected only at small distances where the density gradient is rather small. The resulting local mass density is $0.0099 M_{\odot} \mathrm{pc}^{-3}$.

The kinematics of the dark halo are assumed identical with those of the stellar halo: no rotation, $\left(\sigma_{U} / \sigma_{V} / \sigma_{W}\right)=$ $(131 / 106 / 85) \mathrm{km} \mathrm{s}^{-1}$.
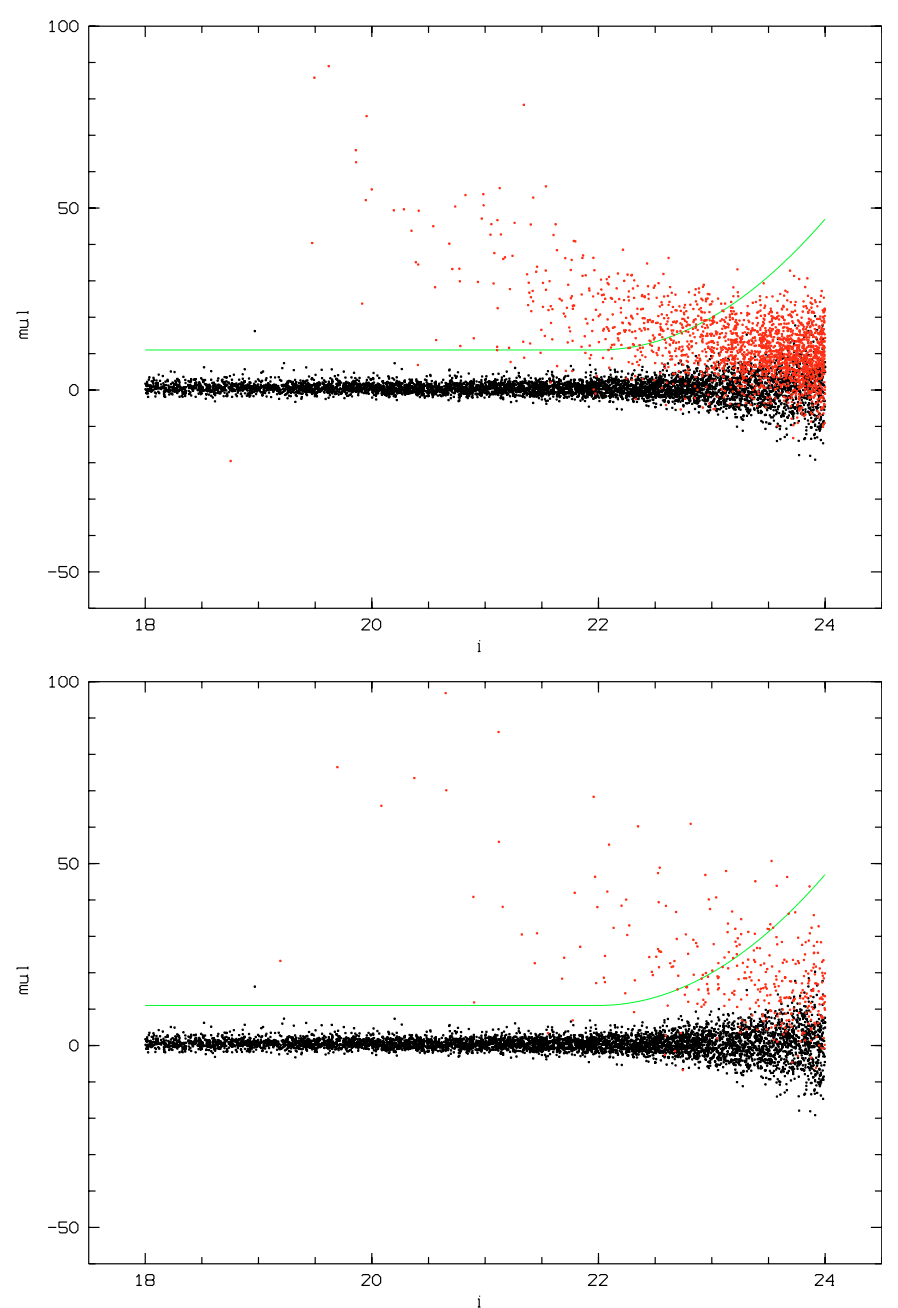

Fig. 6. $I$ vs. $\mu_{l}$ plot for simulated data in the VIRMOS field. Halo white dwarfs in red. Top: halo age $12 \mathrm{Gyr}$, white dwarf mass $0.7 M_{\odot}$. Bottom: $14 \mathrm{Gyr}, 0.8 M_{\odot}$.

Models have been generated using two initial mass functions according to Chabrier (1999): IMF1 has white dwarf mass $0.8 M_{\odot}$ and IMF2 has $0.7 M_{\odot}$. For both IMFs we got the resulting white dwarf luminosity function after 12 or $14 \mathrm{Gyr}$ from Chabrier (2002). Actually, the luminosity distribution of the halo white dwarfs observable in our survey peaks around $M_{v}=16.3$ for IMF2 after $12 \mathrm{Gyr}$ and goes as faint as $M_{v}=17.3$ for IMF1 after 14 Gyr. Most colours range within $1.0<$ $V-I<1.5$. At such colours, the magnitude limit in the $V$ filter is not an issue.

Monte Carlo simulations of dark halos fully made of white dwarfs according to the above mass and luminosity scenarios have been produced for magnitude, colour and proper motion observations in our two fields. The resulting proper motion versus $I$ plots for two extreme models are given in Fig. 6. They are over plotted on the distribution of the three standard populations as predicted by the Besançon model. The upper envelope of observed proper motions as established in Sect. 3.1 and Fig. 4 is also over plotted in green. The constraint brought by not detecting proper motion objects above this envelope is now estimated by simply counting the red points above. 
Table 2. Number of simulated halo white dwarfs above the green limits in Figs. 5 and 6 under different modelling hypotheses in both observing fields. Average detection efficiencies are also given. Model labels indicate the adopted halo age (first number), and IMF (1) or (2) (see text).

\begin{tabular}{crrrr}
\hline \hline Model & $N_{\text {vir }}$ & $\left\langle\mathrm{ef}_{\text {vir }}\right\rangle$ & $N_{\text {SA57 }}$ & $\left\langle\mathrm{ef}_{\mathrm{SA} 57}\right\rangle$ \\
\hline $12(1)$ & 33.6 & 0.84 & 18.5 & 0.67 \\
$12(2)$ & 44.8 & 0.85 & 25.8 & 0.68 \\
$14(1)$ & 13.1 & 0.80 & 6.3 & 0.66 \\
$14(2)$ & 22.4 & 0.82 & 11.0 & 0.65 \\
\hline
\end{tabular}

\subsection{Estimating dark halo fraction}

The number of model predicted halo white dwarfs expected in each field in the magnitude, proper motion volume probed by our survey, above the limits discussed in Sect. 3.1, is given in Table 2 for each halo hypothesis. Simulations were performed in enlarged fields to secure sufficient statistics. The number of expected detections used in Table 3 is obtained by scaling the numbers to the actual observation field, then applying the average efficiency factor for this simulation. The average efficiency factors are obtained by assigning each star an efficiency loss factor corresponding to its $I$ magnitude as from the efficiency calibration given in Fig. 3, then averaging this factor over the stars actually predicted by the adopted model inside the observation limits.

The probability $p$ of detecting no candidates in given observing limits when $m$ detections are expected is $\exp (-m)$ (Poisson distribution).

So if the number of expected detections is $N_{\mathrm{d}}$, the maximum percent of the halo fraction (hfr) acceptable under odd probability $p$ is:

$\left.\operatorname{hfr}\left(p, N_{\mathrm{d}}\right)=(100 * \ln (1 / p)) / N_{\mathrm{d}}\right)$.

Table 3 gives the resulting halo fraction under odd probabilities $p=0.05$ and $p=0.36$ i.e. confidence levels $95 \%$ and $64 \%$ respectively.

Expectedly the estimation of halo fraction is modeldependent. The adopted halo age plays the major role, but it also depends on the adopted initial mass function. The limit of the halo age is now well fixed by CMB fluctuation data at about 13.7 gigayears. Thus, using figures based on 14 gigayears sets the safest limit. The result turns out to be robust to possible overestimation of efficiencies at faint magnitudes.

\section{Comparison with other investigations}

Similar investigations have been published by Goldman et al. (2002) based on a specific proper motion survey by the EROS group within $250 \mathrm{sq}$. deg. down to $V=21.5$, and by Nelson et al. (2002) based on a search for proper motion stars in HST frames in a much smaller field $(0.02078 \mathrm{sq}$. deg.) yet much deeper $(V \leq 26.5, V-I \leq 1.62)$. In the following we attempt to reinvestigate the two data sets using the approach we had with our own data. That is: identify a volume where halo
Table 3. Maximum halo fraction (percent) in the form of ancient white dwarfs compatible with zero effective detection, given the number of expected detections $\left(N \mathrm{~d}_{\ldots} ..\right)$. Numbers are now scaled to actual field size. Halo fractions are given at $95 \%$ and $64 \%$ confidence levels.

\begin{tabular}{crrrrr}
\hline \hline Model & $N \mathrm{~d}_{\text {vir }}$ & $N \mathrm{~d}_{\text {Sa57 }}$ & $N_{\text {tot }}$ & $\mathrm{hfr}_{95}$ & $\mathrm{hfr}_{64}$ \\
\hline $12(1)$ & 28.29 & 12.40 & 40.69 & 7.4 & 2.5 \\
$12(2)$ & 37.95 & 17.54 & 55.49 & 5.4 & 1.8 \\
$14(1)$ & 10.51 & 4.16 & 14.66 & 20.4 & 7.0 \\
$14(2)$ & 18.41 & 7.15 & 25.56 & 11.7 & 4.0 \\
\hline
\end{tabular}

white dwarfs if they exist would be the only significant contributors to the statistics. In the conclusions we will combine the three results.

\subsection{EROS white dwarf search}

The EROS group conclusion is that they exclude halo mass fractions larger than $5 \%$ in the form of ancient white dwarfs. The MACHO/HST group concludes that some of the white dwarfs they have detected "may be members of the halo". In order to combine these results with our own, we re-evaluate the constraint brought by each data set in an homogeneous way. That is, based on our modelling of known stellar populations, we delimitate a region of the magnitude proper motion space where no stars are expected except for halo white dwarfs. And no candidate is found.

Then we compute our own model prediction for a hundred percent white dwarf halo within this limit, since there is no detection in any of the three samples.

In Goldman et al. (2002) the limits of the zero candidate volume are defined as:

$70 \operatorname{arcsec}^{-1}<\mu<600 \operatorname{arcsec}^{-1} \operatorname{cen}^{-1}$

$V<22$

$H_{V}>22.5 ; H_{V}=V+5 \log (\mu / 100)+5$

where

$H_{V}=V+5 \log (\mu / 100)+5$.

The detection efficiencies have been estimated by Goldman (private communication). It degrades from 0.92 at magnitude 16 down to 0.29 at magnitude 21 . Following the same procedure as in the case of our own data each predicted star is assigned a weight equal to the efficiency corresponding to its magnitude. Then the number of expected detections is simply the sum of the weights of predicted white dwarfs.

\subsection{MACHO/HST white dwarf data}

Nelson et al. (2002) used two epoch exposures of the Wide Field Planetary Camera in the so-called Groth-Wesphal strip. In this $74.8 \mathrm{arcmin}^{2}$ survey complete to $V=26.5$ they identify a series of high proper motion objects out of which 5 are considered as strong white dwarf candidates. That is, according to the authors, about three more than expected from standard 


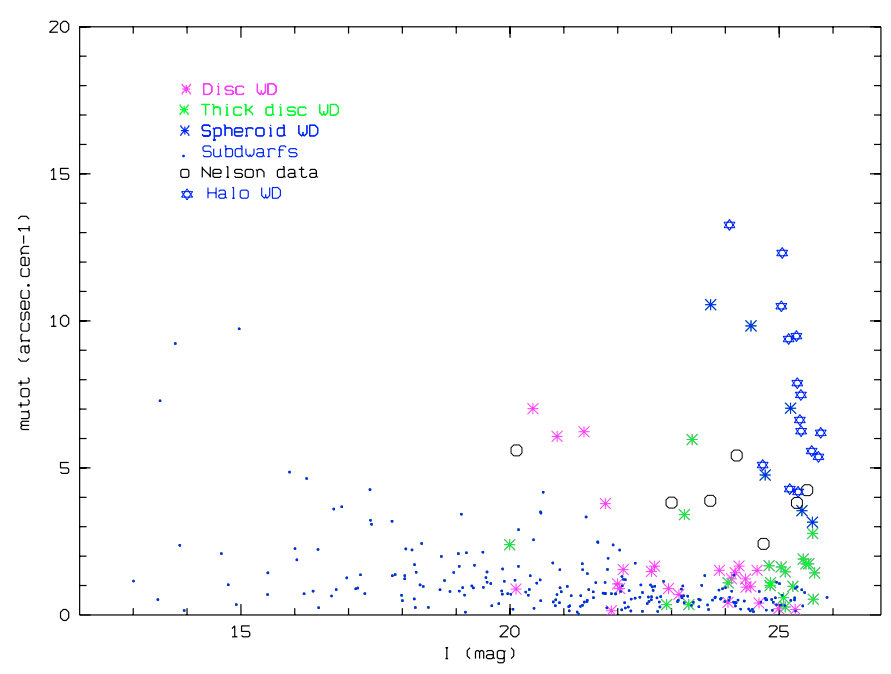

Fig. 7. $|\mu|$ versus $I$ distribution of the HST observations Nelson et al. (2002) compared to model predictions. Model predictions for standard populations are amplified by 10 .

disc, thick disc and spheroid populations. They tentatively interpret their findings as follows: "Possibly, the excess cannot be explained without invoking a fourth galactic component” actually a white dwarf dark halo. Yet they admit that this conclusion is rather model-dependent.

Indeed our simulations for the HST field (Fig. 7) confirm that some of their observed white dwarfs have magnitudes and proper motions compatible with being halo members (They are also compatible with being thick disc or spheroid members). We also confirm that the detected objects outnumber what can be accounted for from standard populations: it is necessary to amplify white dwarf model predictions by at least a factor of 5 to produce a number of thick disc and/or spheroid white dwarf members.

But our simulation shows also that any white dwarf halo model capable of placing a few stars at this place in the magnitude/proper motion diagram would at the same time produce many more detectable stars with higher proper motions where there would be no possible confusion with standard populations. Among the models we tried, those capable of producing one halo white dwarf with $I<25.5$ and total proper motion smaller than 6 arcsec per century produce at least 10 proper motions larger than this limit where Nelson's data has none. So the interpretation of Nelson's stars as halo white dwarf is quite unlikely.

We infer from the Nelson et al. (2002) observations that in a volume within which a full white dwarf halo would put 9 or 10 objects, they detect none. These figures will be combined with others in the next section.

One should also remember that another search based on very deep HST observations produced negative results (Ibata et al. 1999; Richer 2002).

In Table 4 we replicate the estimation presented in Sect. 3.3 incorporating the additional constraints from Nelson et al. (2002) and Goldman et al. (2002).

With EROS data alone (Goldman et al. 2002) this estimation would have produced a maximum halo fraction of $11.2 \%$ (0.95 confidence level) under IMF1 and 6.4\% under IMF2.
Table 4. Same as Table 3 combining data analysed in this paper $\left(N \mathrm{~d}_{\mathrm{C}}\right)$, Nelson's $\left(N \mathrm{~d}_{\mathrm{N}}\right)$ and Goldman's $\left(N \mathrm{~d}_{\mathrm{G}}\right)$. Models are 14.1 (top) and 14.2 (bottom).

\begin{tabular}{rrrrrr}
\hline \hline$N \mathrm{~d}_{\mathrm{C}}$ & $N \mathrm{~d}_{\mathrm{N}}$ & $N \mathrm{~d}_{\mathrm{G}}$ & $N \mathrm{~d}_{\text {tot }}$ & $\mathrm{hf}_{95}$ & $\mathrm{hf}_{64}$ \\
\hline 14.7 & 9 & 26.8 & 49.3 & 6.1 & 2.1 \\
25.6 & 10 & 46.8 & 80.0 & 3.7 & 1.3 \\
\hline
\end{tabular}

With the same data and an IMF close to IMF2 the limit obtained by the authors is $5 \%$ producing a sharper result. The discrepancy comes from slightly different IMF and halo parameters. For the sake of robustness, we used always the most conservative hypotheses. Had we used exactly the same modelisation, it would make the halo fraction even smaller. It would be tempting to combine these results also with the constraint brought by the volumes explored by Flynn et al. (2001) using Luyten's LHS and NLTT proper motion surveys. However the compleness of this survey at faint magnitudes is not well established beyond Luyten's $R$ magnitude 18 . There is no possibility to directly calibrate detection efficiency on Luyten's plates and the indirect calibration used by Flynn et al. (2001) is subject to biases. So we can only notice that constraints brought by Luyten's data strengthen our conclusions without being able to make it a quantitative statement.

\section{Conclusions}

Three deep proper motion surveys produce zero halo white dwarfs within observational limits where a full white dwarf halo would exhibit between 49 and 80 such objects.

This is in obvious conflict with the conclusions of two investigations based on less deep observations (Oppenheimer et al. 2001; Mendez 2002). Although exploring comparable or larger galaxy volumes, surveys at brightest magnitudes cannot safely isolate halo stars. In the two quoted references, the claimed halo white dwarfs have proper motions quite compatible with being thick disc stars. The diagnosis of halo has been rejected by many different sources under different approaches: Reylé et al. (2001), Reid et al. (2001), Silvestri et al. (2002), Torres et al. (2002), Flynn et al. (2003). All support the idea that the thick disc is the most likely explanation.

So the convergence of existing data is quite clear: whenever ancient halo white dwarfs have been searched for under observing conditions where no room was left for confusion with existing standard populations, there were none found. The combined constraint from existing investigations pushes the maximum halo fraction below $4 \%$ at the $95 \%$ confidence level and below $1.3 \%$ (64\% confidence).

This direct observational evidence is consistent with arguments based on the overproduction of carbon relative to oxygen that would accompany the evolution of halo WD precursors (Brook et al. 2003). The only way to keep some limited room open for this hypothetic contributor to the Dark halo baryons would be to adopt Chabrier's IMF1, that is to assume that most dark halo white dwarfs have masses about $0.8 M_{\odot}$. Yet according to Chabrier (1999), the progenitors of such high mass white dwarfs would return more processed gas into the interstellar 
medium per unit mass of white dwarfs produced. So they would contribute more efficiently to the early carbon enrichment.

This implies that if baryonic dark matter is present in galaxy halos, it is not, or is only marginally in the form of faint hydrogen white dwarfs.

Acknowledgements. This research work was partially supported by the Indo-French Centre for the Promotion of Advanced Research (IFCPAR)/Centre Franco-Indien pour la Promotion de la Recherche Avancée (CEFIPRA), New Delhi (India).We thank Gilles Chabrier for providing his models in advance of publication and for useful advice all along this investigation. We also thank David Graff, Bertrand Goldman and an anonymous referee for several constructive remarks.

\section{References}

Afonso, C., Albert, J. N., Andersen, J., et al. 2003, A\&A, 400, 951 Alcock, C., Allsman, R. A., Alves, D., et al. 1997, ApJ, 486, 697 Aubourg, E., Bareyre, P., Brehin, S., et al. 1993, Nature, 365, 623 Bertin, E., \& Arnouts, S. 1996, A\&AS, 117, 393

Brook, C. B., Kawata, D., \& Gibson, B. K. 2003, MNRAS, 343, 913 Chabrier, G. 1999, ApJ, 513, L103

Chabrier, G., Brassard, P., Fontaine, G., \& Saumon, D. 2000a, ApJ, 543, 216

Chabrier, G., Baraffe, I., Allard, F., \& Hauschildt, P. 2000b, ApJ, 542, 464

Chabrier, G. 2002, private communication

Charlot, S., \& Silk, J. 1995, ApJ, 445, 124
Flynn, C., Sommer-Larsen, J., Fuchs, B., Graff, D. S., \& Salim, S. 2001, MNRAS, 322, 553

Flynn, C., Holopainen, J., \& Holmberg, J. 2003, MNRAS, 339, 817

Goldman, B., Afonso, C., Alard, Ch., et al. 2002, A\&A, 389, L69

Ibata, R. A., Richer, H. B., Gilliland, R. L., \& Scott, D. 1999, ApJ, 524, L95

Ibata, R., Irwin, M., Bienaymé, O., Scholz, R., \& Guibert, J. 2000, ApJ, 532, L41

Le Fèvre, O., Mellier, Y., McCracken, H. J., et al. 2004, A\&A, 417, 839

McCracken, H. J., Radovich, M., Bertin, E., et al. 2003, A\&A, 410, 17

Mendez, R. A. 2002, A\&A, 395, 779

Nelson, C. A., Cook, K. H., Axelrod, T. S., Mould, J. R., \& Alcock, C. 2002, ApJ, 573, 644

Oppenheimer, B. R., Hambly, N. C., Digby, A. P., Hodgkin, S. T., \& Saumon, D. 2001, Science, 292, 698

Reid, I. N., Sahu, K. C., \& Hawley, S. L. 2001, ApJ, 559, 942

Reylé, C., Robin, A. C., \& Crézé, M. 2001, A\&A, 378, L53

Richer, H. B. 2002, in The Dark Universe, Matter, Energy and Gravity [arXiv: astro-ph0107079]

Robin, A. C., Reylé, C., Derrière, S., \& Picaud, S. 2003, A\&A, 409, 523

Ruiz, M. T., \& Bergeron, P. 2001, ApJ, 558, 761

Saumon, D., \& Jacobson, S. B. 1999, ApJ, 511, L107

Silvestri, N. M., Oswalt, T. D., \& Hawley, S. L. 2002, AJ, 124, 1118

Stetson, P. B. 1987, PASP, 99, 191

Torres, S., Garcia-Berro, E., Burkert, A., \& Isern, J. 2002, MNRAS, 336, 971 